\title{
Atividade física na fase da meia- idade: motivos de adesão e de continuidade
}

Andréa Krüger Gonçalves ${ }^{1}$, Cátia Pereira Duarte ${ }^{2}$, Cristiane Leite dos S:

\section{Resumo}

A pesquisa realizada tem por objetivo identificar os motivos que conduzem pessoas de meia-idade à participarem de atividades físicas, abordando pontos variados para auxiliar na compreensão de atitudes (expectativas, motivações, resultados, impedimentos). Realiza-se uma investigação social exploratória baseada numa metodologia qualitativa com 60 pessoas ativas. Os resultados permitem a identificação dos motivos que conduzem pessoas de meia-idade (com mínima experiência) a participarem de atividades físicas, porém reafirmam a necessidade de mais investigações sobre a influência social e o comportamento ativo ou sedentário nas diferentes fases de vida.

Unitermos: atividade física, meia-idade, motivos de adesão, motivos de continuidade

\section{Abstract}

Physical Activity In The Middle-Age Phase: Adherence And Continuity Reasons

The aim ofthis research is to identify the reasons that drive the middle-age people to the perform physical activities and to board varied points that assist in the comprehension of the attitudes (expectations, motivations, results, impediments). This study is an exploratory social investigation based in a qualitative methodology with 60 active people. The results allow the Identification of the reasons that drive people with more than four life decades (with minimum experience) practice physical activities, but more investigation is necessary about social influence and the active or sedentary behavior in different life phases.

Keywords: physical activity, middle-age, adherence reasons, continuity reasons

\section{Introdução}

Os estudos na área de Educação Física relacionados à meia-idade são incipientes, porém atualmente há uma tendência prevalecente de associar-se atividade fí-

sica com qualidade de vida, a qual não era verificada anteriormente Atualmente sabe-se de forma tão evidente. A que chegar à velhice seia-se no fato de que será vivenciado por pessoas de meia-idade muitas pessoas. $A$ praticantes de algum tipo procura por de atividade física atividades físicas na acreditam na melhoria de meia-idade pode ser suas condições de vida no um reflexo da tempo presente e também ${ }_{\text {no futuro (ou seja, na fase }}$ preocupação no da terceira idade). Se- sentido de preparação gundo Hayfiick (1997), a para uma fase da expectativa de vida daterceira idade com população em geral qualidade aumentou nas últimas décadas e os idosos representam uma parcela representativa na população total. $\mathrm{O}$ contato com pessoas idosas é um fato cada vez mais constante na vida comum e, se anteriormente pensava-se que a maioria não iria alcançar a velhice, atualmente 
sabe-se que este período de vida será vivenciado por muitas pessoas (em países desenvolvidos de forma mais característica). A procura por atividades físicas na meia-idade pode ser um reflexo da preocupação, com os anos subseqüentes, no sentido de preparação para uma fase da terceira idade com qualidade.

O ano de 1999 foi considerado o Ano Internacional do Idoso e uma das pretensões da 'International Society for Aging and Physical Activity' relacionava-se (e relaciona-se) a ação de reconhecer a meia-idade como uma fase de transição para o envelhecimento ativo, bem como desenvolver mais canais de comunicação entre as gerações. Entre os objetivos mais divulgados destacam-se: melhoria da saúde, segurança e manutenção das pessoas idosas; introdução de imagens sociais novas e apropriadas para a terceira-idade (desmistificação de preconceitos); organização de estruturas de trabalho mais flexíveis e de oportunidades educacionais formais e informais.

A meia-idade e a terceira-idade constituem fases de vida intrinsecamente relacionadas (assim como toda a trajetória de vida). Os estudos relacionados à meia-idade na área da Educação Física podem auxiliar na compreensão deste período de vida a partir da identificação dos objetivos e dos fatores determinantes do envolvimento em atividade física, os quais podem estar relacionados à alguns aspectos relativos da terceira idade: aposentadoria, independência dos filhos, rotina, vida sedentária, entre outros (de acordo com Troll, 1982 e Gonçalves, 1999). Pesquisas com pessoas na meia-idade podem ser também uma forma de contribuir nos estudos sobre envelhecimento.

As fases da meia-idade e da terceira idade são conceituadas como um período de vida, compreendendo uma estrutura que considera o fator tempo e a interação dos fatores biológicos, ambientais, sociais e comportamentais, de acordo com Bromley (1990). Deste modo, cada um destes períodos é definido tanto pelo seu aspecto cronológico, como pelas suas outras características. Bromley sugere a seguinte estrutura para operacionalizar as fases de vida adulta:

\begin{tabular}{|c|c|c|}
\hline $\begin{array}{l}\text { Estágio } \\
\mathbf{n}^{\circ}\end{array}$ & $\begin{array}{l}\text { Idade } \\
\text { em anos }\end{array}$ & Título \\
\hline 1 & $18-21$ & $\begin{array}{l}\text { transição para } \\
\text { independência adulta }\end{array}$ \\
\hline 2 & $22-25$ & Início da idade adulta \\
\hline 3 & $26-40$ & meio da idade adulta \\
\hline 4 & $41-54$ & final (última) idade adulta \\
\hline 5 & $55-60$ & $\begin{array}{l}\text { transiçāo para (pré) } \\
\text { aposentadoria }\end{array}$ \\
\hline 6 & $61-65$ & (pré) aposentadoria \\
\hline 7 & $66-75$ & Idoso-jovem \\
\hline 8 & $76-85+$ & Idoso \\
\hline 9 & $86-120$ & Idoso-idoso \\
\hline 10 & - & estágio terminal \\
\hline
\end{tabular}

De acordo com esta estrutura a fase da meia-idade compreenderia os estágios 4 e 5 , enquanto a fase da terceira-idade iniciaria no estágio 6 . Simões (1994) afirma que o envelhecimento é classificado pela Organização Mundial da Saúde em quatro estágios: meia-idade (45 a 59 anos), idoso (60 a 74 nos), ancião (75 a 90 anos) e velhice extrema (90 anos em diante), seguindo a mesma orientação de Bromley. E importante ressaltar que o corte etário da Organização das Nações Unidas (ONU) é de 65 anos para delimitar a porção idosa de uma população nos países desenvolvidos, mas que nos países em desenvolvimento (como o Brasil) é de 60 anos, devido à menor expectativa de vida, conforme salientam Gavião e Jacquemin (1997). 
O propósito deste estudo é procurar compreender a concepção de pessoas de meia-idade sobre atividade física, tentando estabelecer uma conexão com as perspectivas de envelhecimento. De Rose (apud Centurião Filho, 1998) faz uma retrospectiva sobre o valor da atividade física, salientando que na década de 60 o exercício era visto como um agente na prevenção, tratamento e reabilitação das patologias cardiovasculares; nas décadas de 70 e 80 era a busca do condicionamento físico; atualmente, nos anos 90, valorizase a caminhada e a sua relação com o objetivo de qualidade de vida. Porém, a prática de atividades físicas da maioria da população é restrita aos movimentos do cotidiano e aos do trabalho.

\section{Atividades físicas na fase da meia-idade}

Segundo Brehm (apud Krüger, 1997) os objetivos dos idosos a partir da realização de atividades físicas referem-se à saúde, ao aprimoramento estético e à integração social, podendo-se admitir que este tipo de atividade é imprescindível ao seu bemestar. Porém, de acordo com Matsudo et. ai. (1996) há um baixo nível de aptidão física das populações ao redor do mundo e aumento da prevalência de mortalidade precoce causada por enfermidades da civilização, demonstrando como o estilo de vida sedentário pode ser maléfico para o indivíduo e prejudicial à sociedade. Infelizmente, as sociedades em estágios industriais ou pósindustriais trouxeram uma drástica redução na atividade cotidiana, levando a um quadro geral de hipocinesia (redução de movimentos) e aumento proporcional de moléstias como cardiopatias, diabetes, lombalgias e obesidade, entre outras. Por outro lado, as pesquisas em Medicina do Exercício, e de uma forma mais abrangente nas Ciências do Esporte, têm demonstrado que o estilo de vida ativo tem um impacto positivo em muitos aspectos de saúde individual e coletiva nas diversas fases da vida. Dentre os fatores comportamentais mais associados à saúde, des- tacam-se os hábitos alimentares, o controle do 'stress' e, especialmente, a atividade física regular. Em seu estudo, Matsudo demonstra que mesmo pessoas sedentárias até os 40 anos, podem passar a adotar um estilo ativo de vida e ter um ganho médio de dois anos e meio na expectativa de vida.

Um outro dado ressaltado por Matsudo refere-se às estimativas de epidemiologistas americanos de que 250000 mortes por ano podem ser atribuídas à inatividade física e que 32400 vidas por ano poderiam ser salvas se houvesse um modesto incremento na atividade física. No Brasil não existem dados estatísticos suficientes da população sobre a porcentagem de pessoas sedentárias e fisicamente ativas. Dados epidemiológicos da atividade física e promoção da saúde sobre a população brasileira são escassos, bem como as iniciativas de programas de intervenção.

Segundo Faria Júnior (1999), a partir dos anos 80 tem sido amplamente divulgado pela mídia que mais da metade de todas as pessoas do mundo com idade de 60 anos vive nos países em desenvolvimento. Existe também uma previsão de que até 2025 três quartos da população idosa no mundo estarão vivendo em países menos desenvolvidos. Resultados de uma pesquisa, recentemente realizada pela Fundação do Sistema Estadual de Dados de São Paulo (SEADE), revelam que o Brasil entrará no próximo século com a maior população juvenil de sua história, fruto de um período de alta fecundidade registrado no início dos anos 80 . Tais fatos produzem uma dupla demanda: a do grupo de crianças e jovens com necessidades específicas (aumento da necessidade de aprimoramento da educação e da erradicação das doenças infecto-contagiosas) e o segundo com outras exigências sociais e de saúde. Os problemas de saúde das pessoas idosas caracterizam-se sobretudo pela prevalência de doenças crônico-degenerativas, requerendo intervenções custosas e uso de complexas tecnologias. Deste modo, nos países em desenvolvimento exis- 
te um dilema em termos de políticas públicas porque há escassos recursos governamentais para atender concomitantemente as necessidades dos idosos e as demandas dos jovens.

Os estudos sobre envelhecimento e atividade física têm propiciado conhecimentos interessantes sobre esta temática. Segundo Gonçalves (1999), muitos são os autores que relacionam os beneficios sociais, psicológicos e físicos da atividade física para os indivíduos idosos. O'Brien Cousins (citada por Gonçalves) afirma que os beneficios conhecidos da atividade regular evidenciam que a magnitude da atividade física e do exercício tem sido retratada como um 'recurso de sobrevivencia para os adultos idosos, relacionado a autoeficiência. Entretanto, apesar dos benefícios a maior parte da população idosa não participa regularmente de atividades físicas.

Quanto à participação na atividade física na terceira idade, existem evidências que a causa pode estar no auto direcionamento relacionada a autoeficiência que conduziria a um aumento de motivação e necessidade (contínua) de mais atividade. Porém, o ponto importante é que a pessoa assume uma atitude de acordo com a sua necessidade de auto-realização e auto-eficiência. Contudo, não se pode esquecer da influência dos fatores culturais e sociais que (muitas vezes) impedem essa percepção de senso de eficiência, impedindo uma mudança de comportamento que possibilitaria o auto-direcionamento para a busca de eficiência.

De acordo com O'Brien Cousins e Keating (1995), têm sido desenvolvidas diferentes concepções para indicar quais os fatores que influenciam o nível de atividade das idosas. Uma delas refere-se aos benefícios de saúde como resultado (apesar de muitas perceberem que os fatores de risco do exercício superam os benefícios). Outra concepção é que os indicadores do estilo de vida como ciclo, idade, educação e ambiente social determinam o comportamento ativo no envelhecimento. Há muitas referências sobre as causas e as conseqüências da atividade física na terceira idade, porém são necessários mais estudos para obter-se resultados mais conclusivos ainda não obtidos.

Faria Jr. (1999) cita vários estudos que explicitam a relação positiva entre o envelhecimento e a atividade física. Entre estes, destacam-se os estudos de revisão de Dishmam, demonstrando a redução do risco da depressão com o aumento do exercício. Este fato é corroborado por Farmer et al., Weyerer e Kupfer, quando afirmam que em indivíduos sedentários há um aumento relativo de sintomas depressivos. Para Berger (1989), a atividade é acompanhada por uma redução na ansiedade, depressão e irritação, os quais são os sintomas psicológicos mais comuns do 'stress'. Perlmutter e Hall (1992) destacam a importância do exercício ou da atividade física na manutenção de uma qualidade de vida satisfatória e afirmam que a ausência da atividade física pode ser responsável por grande parte da 'deterioração' que acompanha o envelhecimento.

A corrente teórica conhecida como "Life-span" ou Curso de Vida, uma das mais influentes na emergente área da Psicologia do Envelhecimento, deu origem a um modelo sobre a velhice noral denominado de 'velhice bem-sucedida. Tal modelo é baseado numa perspectiva de desenvolvimento de acordo com as variáveis do contexto. Okuma (1999) comenta que a Educação Física, enquanto área de conhecimento e de atuação profissional, deveria ter mais conhecimentos sobre os avanços da Gerontología e da Psicologia do Envelhecimento para colaborar na ampliação e na atualização da visão sobre o envelhecimento, abandonando a imagem estereotipada do velho e da velhice.

Baseado nos últimos estudiosos torna-se possível afirmar a necessidade de incentivar um padrão de vida ativo. Para tanto, os profissionais da área da Educação Física devem estudar temáticas relacionadas com o cotidiano das pessoas com as quais trabalham, para que se possa ter uma me- 
lhor compreensão dos fatores relacionados com a prática de atividades físicas. Os benefícios desta adesão são reconhecidos no meio acadêmico, porém é preciso descobrir formas de incentivo ao comportamento ativo através de estudos sobre concepções das pessoas envolvidas.

\section{Objetivos da pesquisa}

O objetivo principal deste estudo é investigar os motivos que conduzem pessoas de meia-idade à participarem de atividades físicas. Entre os objetivos específicos destacam-se a identificação das expectativas, das motivações, dos resultados e dos impedimentos destas pessoas quanto à atividade física, procurando compreender as suas atitudes. Um objetivo adicional é a identificação dos motivos de escolha de diferentes locais para a prática de atividade física (se caminhada em via pública ou ginástica em academia). Com esta pesquisa pretende-se verificar se pessoas de meiaidade optam pela atividade física pela sua relação com o tempo presente e tempo futuro (na fase da terceira idade).

\section{Metodologia da pesquisa}

Este trabalho baseia-se numa pesquisa qualitativa social do tipo exploratório realizada com 60 pessoas entre 40-60 anos ${ }^{4}$ participantes de atividades físicas diferenciadas de acordo com dois grupos: grupo caminhada (30 pessoas de ambos os sexos) e grupo 'ginástica' (30 pessoas de ambos os sexos). O instrumento utilizado é o questionário pela sua obtenção de resultados com objetividade (conforme Cervo, 1986), além da facilidade de aplicação e da possibilidade de anonimato dos respondentes.

Triviños (1987) afirma que um estudo exploratório permite suscitar problemas de pesquisa, delimitando uma teoria. O objetivo deste tipo de trabalho é selecionar um número relativamente pequeno de possíveis variáveis ou cate- gorias, a partir de um número extremamente grande, devendo basear-se em amostras relativamente pequenas e cuidadosamente selecionadas, segundo Blalock (1976). Existem situações que implicam estudos de conotação qualitativa (análise de atitudes, motivações, expectativas, valores), conforme Richardson (1989), e uma destas refere-se à pesquisa realizada: compreender aspectos psicológicos relativos a dados que não podem ser coletados de forma completa por outros métodos.

Quanto ao tamanho da amostra num estudo qualitativo, Triviños (1987) ressalta que há pouco empenho para definir operacionalmente as variáveis (elas são descritas e podem ser várias). A determinação da amostra pode utilizar recursos aleatórios, os quais procuram uma espécie de representatividade do grupo maior dos sujeitos que participarão, ou pode decidir intencionalmente de acordo com determinadas condições porque a preocupação maior não é a quantificação da amostragem. Phillips (1974) afirma que as questões relacionadas ao número de sujeitos selecionados, se corresponde ou não a uma amostragem probabilística, dependem dos objetivos perseguidos. Quando o contexto da descoberta é mais importante que o contexto da justificação, uma grande amostragem de probabilidade pode não oferecer vantagens especiais sobe uma pequena amostragem de não-probabilidade. Neste estudo sobre pessoas de meia-idade, a descoberta é mais proeminente que a justificação pelos poucos trabalhos existentes.

Os resultados são analisados de acordo com a análise de conteúdo proposta por Bardin (1977), a qual é comum em estudos qualitativos. Conforme Triviños (1987), este tipo de análise possibilita o estudo das comunicações entre os indivíduos com ênfase no conteúdo 'das mensagens'. A categorização compreende várias etapas de refinamento das respostas dos sujeitos, com o propósito de encontrar um representação do conteúdo destas, ou seja, o 'núcleo de sentido' nas 
respostas das pessoas, para procurar atingir os objetivos do estudo. A análise e a discussão apresentadas baseiam-se em grandes tópicos ou grandes temas, de acordo com a definição de pesquisa social exploratória/piloto qualitativa, sem preocupação de utilização de índices estatísticos. Este posicionamento segue as mesmas proposições de Bosi (1995), que afirma estar preocupada com as declarações dos seus respondentes. Uma aplicação deste tipo de metodologia é utilizada por Bosi (1995) e Gonçalves (1999).

Os grupos da amostra mostram-se distintos principalmente pela prática de atividades físicas com ou sem orientação profissional (respectivamente, academia e via pública: pistas de caminhada construídos paralelamente em avenidas da cidade). Por ser uma pesquisa social qualitativa, o número de participantes é estipulado aleatoriamente, porém com a precaução de que exista o mesmo número de pessoas nos dois grupos.

As perguntas do questionário referem-se aos motivos de adesão à atividade física de pessoas de meia-idade que freqüentam diferentes locais de prática. $\mathrm{O}$ modelo de questionário é organizado em blocos, porém apresenta-se neste trabalho o bloco A e C (o restante é analisado em outro estudo):

- bloco A: dados de identificação correspondendo às perguntas sobre: nome, idade, estado civil, sexo, escolaridade, profissão e ocupação atual de pessoas de meia-idade;

- bloco B: caracterização do dia-a-dia: sono, alimentação, atividade sexual, doença de pessoas de meeia-idade;

- bloco C: definição de atividade física, tipo, freqüência, tempo de participação, motivo de realização e resultados de pessoas de meia-idade;

- bloco D: concepção de envelhecimento e da fase da terceira idade, na opinião de pessoas de meia-idade.
Quanto à metodologia empregada, conforme Gonçalves (1999) enfatiza, é importante salientar que por ser uma pesquisa social exploratória, não existe o propósito de representação de uma população, mas visa apenas a atingir uma compreensão inicial sobre um grupo de pessoas com idade superior aos 40 anos. As assertivas referem-se ao grupo estudado e, em muitos momentos, são comparadas com as afirmações de diversos autores e com as experiências observadas no dia-a-dia. Este estudo pode (como um objetivo maior) evidenciar outras problemáticas para pesquisas futuras, cujas conclusões podem ser generalizadas para outras pessoas. As análises e as discussões referem-se, assim, a este grupo de pessoas que, por ser parte de uma população, espelham a realidade desta, porém generalizações não podem ser realizadas devido à metodologia de estudo empregada.

\section{Apresentação e discussão dos resultados}

\section{Atividade física: motivos e resultados}

Participam do estudo 60 pessoas de meia-idade praticantes de atividade física divididas em dois grupos: grupo 'caminhada (com 30 pessoas) e grupo 'ginástica' (com 30 pessoas). O primeiro grupo realiza caminhadas em pistas públicas da cidade de Pelotas-RS. As características desta caminhada são: duração de no mínimo 30 minutos sem interrupção e num terreno não-aciden-tado (sem elevações e com piso de asfalto ou areia). A extensão é escolhida de acordo com o tempo desejado (caminhadas de 30, 50 ou 60 minutos, por exemplo). Algumas pessoas utilizam toda a extensão da pista (no caso de uma delas, 7 quilômetros), enquanto outras utilizam uma extensão menor e repetem várias vezes o mesmo trajeto para que o tempo de caminhada seja alcançado. As 30 pessoas que praticam caminhada realizam esta atividade em dois locais distintos da cidade de Pelotas, caracterizados por ser uma via pública transformada em pista para 
realização de atividade física ou lazer. O segundo grupo pratica ginástica em academias na mesma cidade, duas ou três vezes por semana com duração de 60 minutos e com exercícios localizados e aeróbicos.

As diferenças entre ambos os grupos de pessoas referem-se ao tipo de atividade física realizada (um grupo caminhada e outro ginástica) e a orientação direta de um profissional para a realização da atividade (existente apenas no grupo 'ginástica'). As pessoas que realizam ginástica em academia têm um profissional da área da Educação Física para orientar a realização dos exercícios durante toda a sua duração, diferentemente das pessoas praticantes de caminhada que não possuem a presença de um professor. De fato, a maioria das

\begin{tabular}{|l|l|l|}
\hline SEXO & masculino & feminino \\
\hline Grupo 'Caminhada' & 05 & 25 \\
Grupo 'Ginástica' & 13 & 17 \\
\hline
\end{tabular}

Tabela 1 - Sexo Grupo 'Caminhada' e Grupo 'Ginástica'

\begin{tabular}{|l|l|l|}
\hline IDADE & 40 a 49 & 50 a 59 \\
\hline Grupo 'Caminhada' & 13 & 17 \\
Grupo 'Ginástica' & 14 & 16 \\
\hline
\end{tabular}

Tabela 2 - Idade Grupo 'Caminhada' e Grupo 'Ginástica' pessoas do grupo caminhada opta por este tipo de atividade pela orientação de um médico ou de pessoas conhecidas que realizam este tipo de atividade, pela facilidade de acesso e de horário, além de não implicar em gasto financeiro com um professor.

\begin{tabular}{|llllll|}
\hline ESTADO CIVIL & casado & solteiro & divorciado/separado & viúvo \\
\hline Grupo 'Caminhada' 22 & 03 & 02 & 03 \\
Grupo 'Ginástica' & 22 & 02 & 06 & & 0 \\
\hline
\end{tabular}

Em ambos os grupos, a

Tabela 3 - Estado Civil Grupo 'Caminhada' e Grupo 'Ginástica' maioria das pessoas são do sexo feminino (25 no grupo 'caminhada' e 17 no grupo 'ginásticatabela 1), enquanto os homens são minoria (05 no primeiro grupo e 13 no segundo grupo). Vários pesquisadores (entre estes O'Brien Cousins e Vertinsky, 1995; 0'Brien Cousins e Keating, 1995; Vertinsky, 1995; Thompson, 1992) demonstram que o sexo feminino possui um padrão de vida mais ativo que o sexo masculino, tanto pelo envolvimento em atividades domésticas e sociais, como pela busca de atividades físicas. Quanto à idade (tabela 2), nos dois grupos a maioria das pessoas têm mais de 50 anos e o restante tem entre 40 a 49 anos. A idade máxima das pessoas em ambos os grupos é de 59 anos. A maioria das pessoas são casadas em ambos os grupos (tabela 3) .

A ocupação dos sujeitos do estudo é variada (tabela 4), pois algumas pessoas mantêm sua vida profissional ativa (vendedores, professores, por exemplo), enquanto outras dedicam-se exclusivamente aos cuidados com a família (donas-decasa) e outras são aposentadas. Estes resultados permitem a identificação de algumas diferenças entre os grupos, principalmente quanto ao maior número de aposentados no grupo 'ginástica' que pode estar relacionado a maior liberdade de horários e a possibilidade de manter compromissos com horários fixos (como no caso da academia). Já no grupo 'caminhada' é evidente o maior número de donas-de-casa, reforçando um dos principais motivos de escolha da caminhada relativo à possibilidade de variação de horário. 


\begin{tabular}{|lllll|}
\hline OCUPAÇÁO & $\begin{array}{l}\text { trabalho } \\
\text { remunerado }\end{array}$ & dona-de-casa & aposentado \\
\hline Grupo 'Caminhada' & 15 & $\cdots$ & 14 & 01 \\
Grupo 'Ginástica' & 19 & 04 & 07 \\
\hline
\end{tabular}

Tabela 4 - Ocupação Grupo 'Caminhada' e Grupo 'Ginástica'
No grupo 'caminhada', a maioria define atividade física como exercício regular ou movimento (figuras 1 e 2), enquanto no grupo 'ginástica menos pessoas têm esta mesma idéia de atividade física como exercício ou como movimento.

Poucas pessoas, em ambos os grupos, consideram atividade física como sinônimo de atividade diária. Um dado interessante relativo à atividade física vincula-se a relação direta entre esta e seu benefício, já que 30\%, aproximadamente, em ambos os grupos mencionam este fato, ou seja, atividade física como sinônimo de benefício.

Entre as atividades físicas realizadas pelas pessoas do estudo, além da caminhada no grupo 'caminhada e da ginástica em academia no grupo 'ginástica, o esporte e o trabalho também são citados como tipos de atividade física em ambos os grupos. Um fato a ser salientado é que algumas pessoas praticantes de caminhada também freqüentam academia. Esta tendência é mais forte no grupo 'ginástica, pois oito pessoas realizam ginástica em academia e também realizam caminhadas. Pode-se afirmar que o grupo 'ginástica tem um envolvimento maior em atividades físicas de uma forma geral. Talvez uma das explicações para esta diferença encontre-se na disponibilidade maior de tempo do grupo 'ginástica, já que neste há mais pessoas aposentadas que no grupo 'caminhada'.

O grupo 'caminhada e o grupo 'ginástica' possuem a mesma freqüência semanal em suas acaba limitando o modo de vida destas pessoas, impedindo um desenvolvimento pleno.

\begin{tabular}{|c|c|c|c|c|c|c|}
\hline Escolaridade & $\begin{array}{l}1^{\circ} \text { grau } \\
\text { incompleto }\end{array}$ & $\begin{array}{l}1^{\circ} \text { gtau } \\
\text { completo }\end{array}$ & $\begin{array}{l}2^{\circ} \text { grau } \\
\text { incompleto }\end{array}$ & $\begin{array}{l}2^{\circ} \text { grau } \\
\text { completo }\end{array}$ & $\begin{array}{l}3^{\circ} \text { grau } \\
\text { incompleto }\end{array}$ & $\begin{array}{l}3^{\circ} \text { grau } \\
\text { completo }\end{array}$ \\
\hline Grupo 'Caminhada' & 10 & 4 & 2 & 4 & 1 & 9 \\
\hline Grupo 'Ginástica' & - & 3 & $\because$ & 8 & 5 & 14 \\
\hline
\end{tabular}

Tabela 5 - Escolaridade Grupo 'Caminhada' e Grupo 'Ginástica' 
Figura 1 - Definição de Atividade Fisica Grupo 'Caminhada'

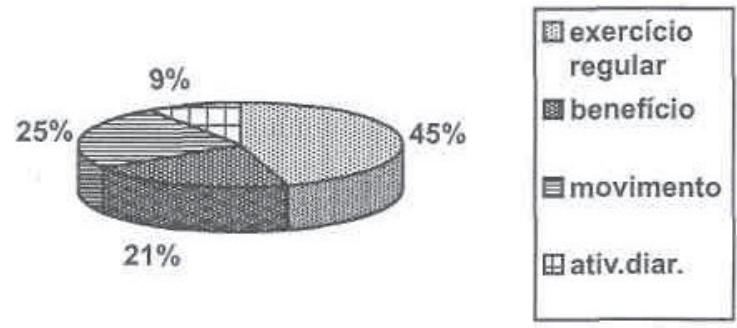

Figura 2 - Definição de Atividade Física Grupo 'Ginástica'
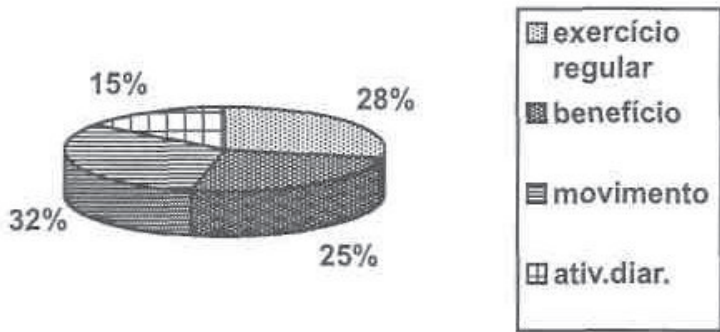

atividades físicas específicas (no caso: caminhada e ginástica) que é de aproximadamente três vezes semanais ou mais. O tempo de participação em atividades físicas, tanto no grupo caminhada como no grupo 'ginástica', varia em torno de um ano. Poucas pessoas confirmam uma prática contínua ao longo das suas vidas. Estas informações corroboram o fato de que a atividade física não faz parte de todo ciclo de vida das pessoas de meia-idade (não é contínua), além de demonstrar a ausência de experiência anterior neste tipo de envolvimento.

As variáveis relacionadas à adesão e à manutenção de atividade física num estudo realizado com indivíduos idosos (Gonçalves, 1998) dependem da percepção de poucas barreiras para a atividade física, de muitos benefícios desta e de alguma sugestão para ação (principalmente proveniente da família, de médico ou de algum meio de comunicação). Os principais fatores associados com a atividade física são a promoção de saúde, a liberação de tensão e os benefícios sociais (McAuley e Rudolph, 1995). As pessoas idosas mostram a concepção de atividade física relacionada à obtenção de benefícios físicos, psicológicos e sociais. Os resultados deste estudo de Gonçalves são condizentes com algumas das concepções colocadas por O'Brien Cousins e Keating (1995) sobre os fatores influenciadores do nível de atividade de sujeitos idosos: os benefícios de saúde como resultado, ou o prazer da participação, ou ainda a necessidade de auto-eficiência.

Hayslip, Weigand, Weinberger, Richardson e Jackson (1996) associam o estilo de vida saudável diretamente à atividade física ou exercício. Os benefícios físicos e emocionais do exercício têm sido documentados e a decisão de iniciar e manter uma atividade física regular é influenciada pela expectativa, assim como pela obtenção destes benefícios. As convicções sobre os benefícios e os gastos potenciais do comportamento de exercício regular podem mudar com o aumento da idade. Deste modo, conforme a idade aumenta, os fatores como saúde debilitada, problemas físicos, isolamento dos outros devido à falta de saúde, aposentadoria, sustento baseado numa única renda fixa e depressão, podem influenciar a motivação das pessoas idosas para que não sejam ativas. Estes fatores podem, entretanto, interferir no início do exercício regular, que pode aumentar o bem-estar ou melhorar o funcionamento cognitivo ou fisiológico. Estes aspectos anteriormente mencionados são verificados nos motivos de adesão mencionados pelas pessoas deste estudo.

Os motivos de adesão à atividade física não variam muito entre pessoas de meia-idade e de terceira idade. O motivo predominante é a saúde do físico e da mente, o qual é reforçado pelo condicionamento físico que também aparece como uma causa para a realização de atividades físicas (figuras 3 e 4). A diferença entre o grupo caminhada e o grupo 'ginástica', quanto ao condicionamento, é diretamente relacionada ao tipo de atividade realizada. No grupo 'ginástica a academia permite (devido a orientação direta de um profissional) o alcance de resultados específicos 


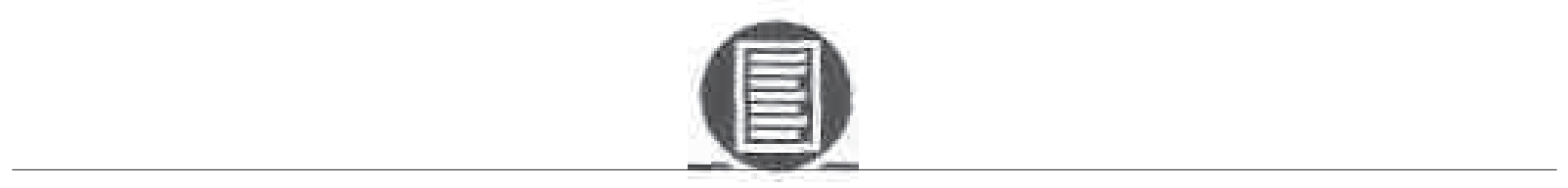

Figura 3 - Motivos de Adesão 'Grupo Caminhada'

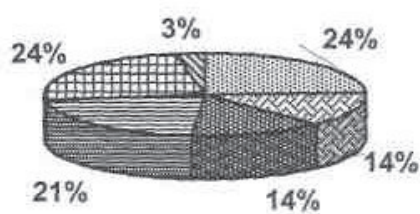

圈 rec.médica

Rmov.natural

달 adeq.idade

目local

agrad.lecon.

\#saúde

Sestética

Figura 4 - Motivos de Adesão 'Grupo Ginástica'

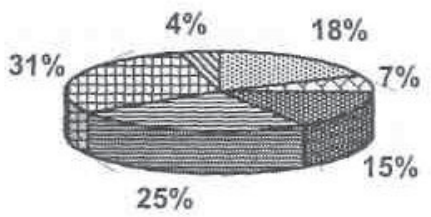

\begin{tabular}{|c|}
\hline 国 rec.médica \\
\hline Qativ.variada \\
\hline 眼adeq.idade \\
\hline $\begin{array}{l}\text { ⿴local } \\
\text { agrad.lecon. } \\
\text { 田saúde }\end{array}$ \\
\hline Mestética \\
\hline
\end{tabular}

(melhoria da resistencia, aumento de força, perda de peso). Apesar do motivo estético ser um dos fatores mais fortes de adesão às atividades físicas, neste estudo isto não pode ser comprovado. No grupo 'ginástica nenhuma pessoa menciona a estética como motivo de adesão, já no grupo 'caminhada doze pessoas apontam esta como fator motivador. A caminhada é uma das atividades físicas mais preconizadas pela mídia (a outra atividade é o esporte) e a estética está sendo diretamente relacionada a sua prática. A ginástica em academia por sua vez não é uma prática incentivada da mesma forma pelos meios de comunicação e uma das explicações é o aspecto financeiro, pois poucas pessoas no Brasil têm condições de freqüentar este espaço.

O motivo da atividade específica (caminhada ou ginástica) é a saúde em ambos os grupos. A recomendação de outras pessoas (médico, colega, propaganda) confirma a preponderância deste fator como adesão. O local de realização da atividade física também é citado, podendo ser agradável ou econômico (especificamente no caso da caminhada). No grupo 'caminhada a naturalidade do movimento é apontada como um motivo específico, no grupo 'ginástica a diversidade de movimento torna-se um fator importante de adesão. A adequação da atividade escolhida à idade é um outro fator mencionado pelas pessoas nos dois grupos.

Os resultados obtidos com a participação em atividades físicas confirmam os motivos de adesão mencionados pelas pessoas, estando diretamente vinculados à realização de exercícios regulares para o desenvolvimento físico e mental: maior disposição, condicionamento físico, diminuição de dores, liberação de tensão e estética vinculada diretamente à perda de peso (figuras 5 e 6). Terry, Biddle, Chatzisarantis e Bell (1997) afirmam que a investigação dos resultados da participação (se físicos ou psicológicos) é necessária porque não se sabe como as pessoas idosas (nem em outras idades) percebem a atividade física. Desta forma, é importante entender os fatores prováveis de serem associados com o envolvimento na atividade física. Os benefícios associados à atividade física pelas pessoas de meiaidade são similares às atitudes mencionadas por idosos nos estudos anteriores: liberação de tensão, referindo-se à atividade física como reparadora de tensão; irritabilidade e ansiedade; promoção de saúde, referindo-se à manutenção da saúde através de intensa atividade física; e benefícios sociais, referindo-se ao exercício como fator de diversão, de socialização e de promoção para melhor uso do tempo livre.

Figura 5 - Resultados 'Grupo Caminhada'

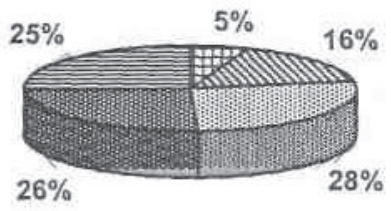

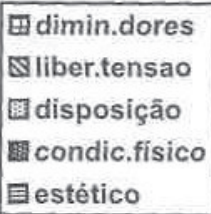




\section{Figura 6 - Resultados} 'Grupo Ginástica'

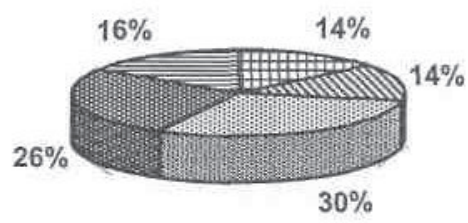

I dimin.dores \&liber.tensão 圆 disposiçao 国 condic.fisico 目estético

Os motivos de continuidade também corroboram os motivos de adesão e os resultados obtidos a partir da prática de caminhada no grupo 'caminhada e da ginástica no grupo 'ginástica' (figuras 7 e 8). As pessoas pretendem continuar as atividades físicas devido ao bem-estar e disposição proporcionados para as atividades cotidianas, podendo ser um meio para propiciar um envelhecimento sem dependência de familiares. No entanto, o grupo 'caminhada' salienta que um motivo para a continuidade na atividade é a melhora efetiva da saúde e estética, maior que o grupo 'ginástica', confirmando os fatores de adesão citados nas respostas anteriores.

Figura 7 - Motivos de Continuidade 'Grupo Caminhada'

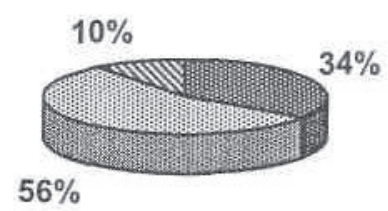

图 bem-estar/disp

국saúde/estética

\$independência

Figura 8 - Motivos de Continuidade 'Grupo Ginástica'

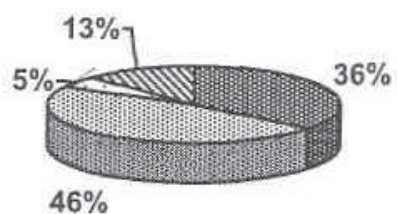

\section{Considerações finais}

Os resultados permitem identificar a concepção de atividade física de um grupo de pessoas de meia-idade. Estas pessoas definem atividade física como exercício regular (movimento) ou trabalho, poucos a consideram sinônimo de atividade diária e grande parte associa seus benefícios como sinônimo de definição. A maioria das pessoas participam de atividades físicas no mínimo há um ano, demonstrando que este tipo de atividade não é uma constante nas suas vidas.

O principal motivo de adesão à atividade $O$ s resultados obtidos física, por parte das com a participação em saúde do físico e da atividades físicas mente. Apesar de existir confirmam os motivos uma diferença básica nos de adesão dois grupos de pessoas de mencionados pelas meia-idade estudados pessoas, vinculados à (com e sem orientação realização de profissional), a melhoria exercícios regulares salientado. As pessoas do para o grupo 'ginástica' desenvolvimento físico salientam benefícios e mental: maior físicos mais específicos, ao disposição, contrário do grupo condicionamento 'caminhada que associa físico, diminuição de de a estética ser um forte dores, liberação de motivo de adesão, neste tensão e estética estudo isto vinculada não é evidenciado. Os diretamente a perda motivos de continuidade de peso mais mencionados são o bem-estar e a disposição, estando diretamente relacionados às atividades de vida diária e a preparação para um envelhecimento sem dependência (já que este fator é um dos principais aspectos referentes ao envelhecimento, conforme Thompson, 1992). 
Krüger (1997) ressalta que os valores, as atitudes, o entendimento e a conduta das pessoas (referindo-se especificamente aos idosos), em relação à cultura física, depende dos sistemas de crenças elaborados e aceitos ao longo de suas experiências. As reações individuais das pessoas em relação à esta cultura física dependem da maneira como cada uma representa, entende e valoriza suas práticas. Da mesma forma, pode-se aplicar tal proposição em relação às pessoas de meia-idade, visto que, a prática de atividades físicas é diretamente relacionada à concepção de vida presente e futura, embora nem sempre tal relação seja explícita. Krüger salienta que é preciso conhecer preliminarmente o sistema de crenças de cada interessado para tornar mais viável a participação em atividades físicas. Os dados desta pesquisa possibilitam o acesso às opiniões de algumas pessoas de meia-idade, permitindo indicar alguns dados quanto à atividade física deste grupo. Os resultados obtidos com a participação em atividades físicas confirmam os motivos de adesão mencionados pelas pessoas, estando diretamente vinculados à realização de exercícios regulares para o desenvolvimento físico e mental: maior disposição, condicionamento físico, diminuição de dores, liberação de tensão e estética vinculada diretamente à perda de peso. A partir desta experiência, molda-se o conceito de atividade física por parte das pessoas de meia-idade como movimento que possibilita grandes benefícios ao corpo humano.

A influência social e a necessidade de ser reconhecido estimulam milhares de pessoas a adotar comportamentos e hábitos mais saudáveis, sendo a preocupação com a saúde uma constante na sociedade atual (de acordo com Nieman, 1999; Manidi e Michel, 2001). A atividade física atua como meio para alcançar idéias mais otimistas sobre o envelhecimento e sobre qualidade de vida, de acordo com Telama (apud Faria Jr., 1999). As doenças diretamente associadas com a saúde afetam a auto-imagem, resultando em novas relações consigo e com os outros. Nas respostas das pessoas de meia-idade é forte a relação com a doença, conforme constata-se nos motivos e resultados da participa- ção em atividades físicas: aumento de disposição ou melhor funcionamento físico.

Os resultados permitem a identificação dos motivos que conduzem pessoas com mais de quatro décadas de vida (a maioria sem ou com mínima experiência) a participarem de atividades físicas. Também possibilitam mostrar que o aumento da expectativa de vida na sociedade atual e a experiência da fase da terceira idade associam-se com esta busca por um padrão de vida ativo, embora esta relação não seja explícita. A influência social com seus estereótipos moldam o comportamento das pessoas em todas as fases de vida e, na meiaidade, os reflexos são evidentes.

São necessários mais estudos que investiguem a percepção e avaliação de temáticas objetivamente ou subjetivamente relacionadas, como atividade física e envelhecimento. As pessoas acreditam que através da atividade física o envelhecimento torna-se um processo mais agradável pela sua compreensão e aceitação, porém a Educação Física precisa valorizar estes resultados através da sua divulgação. Para tanto, é preciso reconhecer que as pessoas possuem necessidades diferenciadas, não podendo determinar os mesmos objetivos à todos. Porém, este conhecimento somente é obtido a partir de trabalhos específicos com populações específicas que estejam baseados em problemáticas do cotidiano da população (neste trabalho, o caso da população de meia-idade).

Os profissionais de Educação Física precisam reconhecer e compreender o potencial educativo da atividade física para desenvolver uma melhor consciência corporal e, consequentemente, melhor qualidade de vida. A estabilidade da saúde, a manutenção da condição física, o contato e a comunicação com outras pessoas, a independência e a autonomia na resolução das tarefas cotidianas são resultados obtidos com a prática da atividade física em todas as fases de vida. Porém, em alguns períodos estes benefícios associam-se no combate às atitudes de isolamento em decorrência das características de vida: idosos, doen- 
tes, deficientes. As atividades físicas adotadas pelas pessoas de meia-idade nesta pesquisa ajudam na manutenção de um estilo de vida saudável, conforme evidencia os resultados deste estudo.

E evidente que existe urna grande preocupação com a manutenção do físico no presente e no futuro (terceira idade) por parte das pessoas com mais de 40 anos, porém há uma forte dissociação entre corpo e mente associada ao 'velhismo'. O aumento da expectativa de vida na sociedade atual e a experiência da fase da terceira idade associam-se com a busca por um padrão de vida ativo, embora esta relação não seja explícita. A influência social com seus estereótipos molda o comportamento das pessoas em todas as fases de vida e, na meia-idade, os reflexos são evidentes. São necessárias mais investigações abordando a fase de vida da meia-idade, a qual tem sido pouco estudada e carece de maiores conhecimentos pelo seu aspecto social (melhor qualidade de vida) como pelo seu aspecto quantitativo (o aumento da expectativa de vida).

\section{Referências Bibliográficas}

BARDIN, L. Análise de conteúdo. Lisboa, Edições 70, 1977.

BEE, H. O ciclo vital. Porto Alegre, Artes Médicas, 1997.

BERGER, B.G. The role of physical activity in the life quality of older adults. In: SPIRDUSO, W.W.; ECKERT, H.M. American Academy of Physical Education: the academy papers: physical activity and aging. Champaign, Human Kinetics, 1989. p.42-57.

BLALOCK, H.M. Introdução à pesquisa social. Rio De Janeiro, Zahar, 1976.

BOSI, E. Memória e sociedade: lembranças de velhos. São Paulo, Companhia das Letras, 1995.

BROMLEY, D.B. Behavioural Gerontology: central issues in the Psychology of Ageing. England-
Chichester, John Wiley \& Sons, 1990.

CENTURIÃO FILHO, AA. A atividade física para o idoso como promoção de autonomia e formação de auto imagem. Caderno Adulto NIEATI, n. 2, 1998.

CERVO, A.L. Metodologia científica. 4.ed. São Paulo, Makrow, 1986.

FARIA JÚNIOR, A.G. Atividades físicas para idosos: um desafio para a educação gerontológica. In: Anais $7^{\circ}$ Congresso de Educação Física e Ciências do Esporte dos Países de Língua Portuguesa. Florianópolis, 1999. p.119-128.

GAVIÃO, A.C.D.; JACQUEMIN, A. Psicanálise e envelhecimento: uma revisão. Mudanças, São Bernardo do Campo, n. 8, p.121-158, 1997.

GONÇALVES, A.K. Expe riência de indivíduos idosos em atividades físicas durante o ciclo de vida e sua influência na atividade física atual. In: Anais VI Congreso de Educación Física e Ciencias do Deporte dos Países de Língua Portuguesa. La Coruña/Espanha, 1998. p.120.

GONÇALVES, A.K. Ser idoso no mundo: o indivíduo idoso e a vivência de atividades físicas como meio de afirmação e identidade social. São Paulo, 1999. 182p. Tese (Doutorado) Instituto de Psicologia, Universidade de São Paulo, 1999. 214p.

HAYFLICK, L. Como e por que envelhecemos. Rio de Janeiro, Campus, 1997.

HAYSLIP, B.; WEIGAND, D.; WEINBERG, R; RICHARDSON, R; JACKSON, A. The development of new scales for assessing health belief model constructs in adulthood. Journal of Aging and Physical Activity, n. 4, v. 4, p.307-323, oct., 1996.

KRUGER, H. A cultura física sob a perspectiva dos idosos. In: Congresso Mundial de Educação Física. Rio de Janeiro, 1997. (texto impresso da palestra conferida)

MANIDI, M.; MICHEL, J. Atividade física para adultos com mais de 55 anos. São Paulo, 
Manole, 2001

MATSUDO, VK.R; ANDRADE, D.R.; MATSUDO, S.M.M; FIGUEIRA JÚNIOR, A.J.; ARAÚJO, T.L.; ANDRADE, E.L.; ROCHA, J.R. Exercício e qualidade de vida. In: $3^{\mathbf{0}}$ Congresso LatinoAmericano 'International Council for Health, Physical Education, Recreation, Sport \& DanceICHPER SD'. Foz do Iguaçu, 1996, p.76-84.

McAULEY, E.; RUDOLPH, D. Physical activity, aging and psychological well-being. Journal of Aging and Physical Activity, USA-Champaign, n. 1,v. 3, p.67-96, jan., 1995.

NIEMAN, D. Exercício e saúde. São Paulo, Manóle, 1999.

O'BRIEN COUSINS, S.; KEATING, N. Life cycle patterns os physical activity among sedentary and active older women. Journal of Aging and Physical Activity, USA-Champaign, n. 4, v. 3, p.340-359, oct, 1995.

O'BRIEN COUSINS, S.; VERTINSKY, PA. Recapturing the physical activity experiences of the oíd: a study of three women. Journal of Aging and Physical Activity, USA-Champaign, n. 2, v. 3, p.146-162, april, 1995.

OKUMA, S.S. Impacto da atividade física sobre a dimensão psicológica do idoso: uma análise sob a ótica da velhice bem-sucedida. In: Anais $7^{\circ}$ Congresso de Educação Física e Ciências do Esporte dos Países de Língua Portuguesa. Florianópolis, 1999. p.111-118.

PERLMUTTER, M.; HALL, E. Adult development and aging. New York, John Wiley \& Sons, 1992.

PHILIPS, B.S. Pesquisa social: estratégias e táticas. Rio de Janeiro, Agir, 1974.

RICHARDSON, RJ. et al. Pesquisa social; métodos e técnicas. 2.ed. São Paulo, Atlas, 1989.

SIMÕES, R. Corporeidade e terceira idade. Piracicaba, UNIMEP, 1994.

TERRY, PC; BIDDLE, S.J.H.; CHATZISARANTIS, N.; BELL, R.D. Development of a test to assess the attitudes of older adults toward physical activity and exercise. Journal of Aging and Physical Activity, n. 2, v. 5, p.111-125, april, 1997.

THOMPSON, P. 'I dont feel old': subjective ageing and the search for meaning in later life. Ageing and Society, England, v. 12, p.23-47, march, 1992.

TRIVIÑOS, A.N.S. Introdução à pesquisa em Ciências Sociais: a pesquisa qualitativa em Educação. São Paulo, Atlas, 1987.

TROLL, L.E. Continuations: adult development and aging. California: Brooks/Cole, 1982.

VERTINSKY, P.A. Stereotypes of aging women and exercise: a historical perspective. Journal of Aging and Physical Activity, USA-Champaign, n. 3,v. 3, p.223-237, july, 1995.

\section{Agradecimentos}

A Escola Superior de Educação Física da Universidade Federal de Pelotas que propiciou o desenvolvimento de estudo, enquanto a primeira autora era professora substituta nesta unidade.

Ao Programa Especial de Treinamento (PET) que possibilitou bolsa de estudos para a aluna Cátia Pereira Duarte, enquanto acadêmica do curso de Educação Física da Universidade Federal de Pelotas.

\section{Notas}

${ }^{1}$ Professora Doutora do curso de Educação Física da Universidade Luterana do Brasil-ULBRA/Canoas.

${ }^{2}$ Licenciada em Educação Física pela Universidade Federal de Pelotas e Bolsista do Programa Especial de Treinamento (PET) durante a graduação.

${ }^{3}$ Acadêmica do curso de Educação Física da Universidade Federal de Pelotas.

${ }^{4}$ Embora, a idade limite para ingresso na fase da meia-idade seja a idade de 45 anos, neste estudo utilizou-se a faixa etária entre 40 a 59 anos. 